\title{
Homoclinic Solutions for a Class of the Second-Order Impulsive Hamiltonian Systems
}

\author{
Jingli Xie, ${ }^{1,2}$ Zhiguo Luo, ${ }^{2}$ and Guoping Chen ${ }^{1}$ \\ ${ }^{1}$ College of Mathematics and Statistics, Jishou University, Jishou, Hunan 416000, China \\ ${ }^{2}$ Department of Mathematics, Hunan Normal University, Changsha, Hunan 410081, China \\ Correspondence should be addressed to Zhiguo Luo; luozg@hunnu.edu.cn
}

Received 16 May 2013; Accepted 18 July 2013

Academic Editor: Juan J. Nieto

Copyright (c) 2013 Jingli Xie et al. This is an open access article distributed under the Creative Commons Attribution License, which permits unrestricted use, distribution, and reproduction in any medium, provided the original work is properly cited.

This paper is concerned with the existence of homoclinic solutions for a class of the second order impulsive Hamiltonian systems. By employing the Mountain Pass Theorem, we demonstrate that the limit of a $2 k T$-periodic approximation solution is a homoclinic solution of our problem.

\section{Introduction and Main Results}

In this paper, we consider the second-order impulsive differential equation

$$
\begin{gathered}
\ddot{q}(t)+V_{q}(t, q)=f(t), \quad t \neq t_{j}, t \in \mathbb{R}, \\
-\Delta \dot{q}\left(t_{j}\right)=g_{j}\left(q\left(t_{j}\right)\right), \quad j \in \mathbb{Z},
\end{gathered}
$$

where $q \in \mathbb{R}^{N}, f \in\left(\mathbb{R}, \mathbb{R}^{N}\right), V_{q}(t, q)=\operatorname{grad}_{q} V(t, q), g_{j}(q)=$ $\operatorname{grad}_{q} G_{j}(q), G_{j} \in\left(\mathbb{R}^{N}, \mathbb{R}^{N}\right)$ for each $j \in \mathbb{Z}$, and the operator $\Delta$ is defined as $\Delta \dot{q}\left(t_{j}\right)=\dot{q}\left(t_{j}^{+}\right)-\dot{q}\left(t_{j}^{-}\right)$, where $\dot{q}\left(t_{j}^{+}\right)\left(\dot{q}\left(t_{j}^{-}\right)\right)$ denotes the right-hand (left-hand) limit of $\dot{q}$ at $t_{j}$. There exist an $m \in \mathbb{N}$ and a $T>0$ such that $0=t_{0}<t_{1}<\cdots<t_{m}=T$, $t_{j+m}=t_{j}+T$, and $g_{j+m}=g_{j}, j \in \mathbb{Z} . V: \mathbb{R} \times \mathbb{R}^{N} \rightarrow \mathbb{R}$ satisfies

(V1) $V(t, q)=-K(t, q)+W(t, q), K, W \in C^{1}\left(\mathbb{R} \times \mathbb{R}^{N}, \mathbb{R}\right)$, and is $T$-periodic in its first variable.

We are mainly concerned with the existence of homoclinic solutions of system (1) and (2). A function $q(t) \epsilon$ $C\left(\mathbb{R}, \mathbb{R}^{N}\right)$ is said to be a (classical) solution of (1) and (2) if $q(t)$ satisfies (1) and (2). A (classical) solution $q(t)$ of (1) and (2) is a homoclinic solution and if $q(t) \rightarrow 0$ as $t \rightarrow \pm \infty$ and $\dot{q}\left(t^{ \pm}\right) \rightarrow 0$ as $t \rightarrow \pm \infty$.

When $\Delta \dot{q}\left(t_{j}\right) \equiv 0, f(t)=0$, and $V(t, q)=(1 / 2)(L(t) q, q)+$ $W(t, q)$, system (1) and (2) reduces to Hamiltonian system

$$
\ddot{q}(t)+V_{q}(t, q)=0, \quad t \in \mathbb{R} .
$$

Rabinowitz [1] studied the existence of nontrivial homoclinic solutions of it.

When $\Delta \dot{q}\left(t_{j}\right) \equiv 0$ and $V(t, q)$ satisfied (V1), system (1), (2) reduces to Hamiltonian system

$$
\ddot{q}(t)+V_{q}(t, q)=f(t), \quad t \in \mathbb{R} .
$$

Izydorek and Janczewska [2] studied the existence of homoclinic solutions of it.

Some classical tools such as some fixed point theorems in cones, topological degree theory, the upper and lower solutions method combined with monotone iterative technique, and variational methods [3-20] have been widely used to get solutions of impulsive differential equations. However, the existence of homoclinic solutions for the impulsive systems is paid little attention. It is well known that the homoclinic orbit rupture phenomenon can lead to chaos, which has been interesting to the mathematicians in recent years [21-26]. In the literature, Coti-Zelati et al. [27] used dual variational methods, and Lions [28] and Hofer and Wysocki [29] employed concentration compactness method, Ekeland's variational principle, that they established the existence of homoclinic solutions of the first-order Hamiltonian systems. Rabinowitz [1] and Izydorek and Janczewska [2] obtained homoclinic solutions of a class of second order Hamiltonian systems as a limit of its periodic solutions.

In recent paper [18], Zhang and Li studied the existence of homoclinic solutions of an impulsive Hamiltonian system 
$q^{\prime \prime}(t)+V_{q}(t, q)=f(t), t \neq t_{j}, t \in \mathbb{R}, \Delta q^{\prime}\left(t_{j}\right)=g_{j}\left(q\left(t_{j}\right)\right), j \in \mathbb{Z}$ as a limit of its periodic solutions. In detail, they obtained the following theorem.

Theorem A (see [18]). Assume that $f \in C\left(\mathbb{R}, \mathbb{R}^{\mathbb{N}}\right) \bigcap$ $L^{2}\left(\mathbb{R}, \mathbb{R}^{\mathbb{N}}\right), g_{j}$ is continuous and m-periodic in $j$, and $V, g_{j}$ satisfy the following conditions:

(H1) $V: \mathbb{R} \times \mathbb{R}^{N} \rightarrow \mathbb{R}$ is continuous differentiable $T$ periodic, and there exist positive constants $b_{1}, b_{2}>0$ such that

$$
b_{1}|q|^{2} \leq-V(t, q) \leq b_{2}|q|^{2}, \quad \forall(t, q) \in[0, T] \times \mathbb{R}^{\mathbb{N}}
$$

$(\mathrm{H} 2)-V(t, q) \leq-V_{q}(t, q) q \leq-2 V(t, q)$ for all $(t, q) \in$ $[0, T] \times \mathbb{R}^{\mathbb{N}}$

(H3) $\lim _{|q| \rightarrow 0}\left(g_{j}(q) /|q|\right)=0$ for $j=1,2, \ldots, m$;

(H4) there exists a $\mu>2$ such that

$g_{j}(q) q \leq \mu G_{j}(q)<0, \quad$ for $q \in \mathbb{R}^{\mathbb{N}} \backslash\{0\}, j=1,2, \ldots, m$,

then the Hamiltonian $\ddot{q}(t)+V_{q}(t, q)=f(t), t \neq t_{j}, t \in$ $\mathbb{R}, \Delta q^{\prime}\left(t_{j}\right)=g_{j}\left(q\left(t_{j}\right)\right), j \in \mathbb{Z}$, possesses at least one nonzero homoclinic solution.

Motivated by papers $[1,2,18]$, in this paper, we synthesize their methods to study the existence of homoclinic solutions of systems (1), (2). In detail, firstly, we introduce the following sequence equations:

$$
q^{\prime \prime}(t)+V_{q}(t, q)=f_{k}(t), \quad t \neq t_{j}, t \in \mathbb{R},
$$

where for each $k \in \mathbb{N}, f_{k}: \mathbb{R} \rightarrow \mathbb{R}^{N}$ is a $2 k T$-periodic extension of the restriction of $f$ to the interval $[-k T, k T]$. Secondly, we study periodic solutions of (2) and (7) by converting the problem to the existence of critical points of some variational structure. finally, we find the homoclinic solutions of (1) and (2) as the limit of the periodic solutions of (2) and (7).

Part of the difficulty in treating (1) and (2) is subjected to the impulsive perturbation which destroys continuities of the velocity and when we apply the Mountain Theorem to prove our main result, we need the constant $\rho, \alpha$ appearing in the theorem to be independent of $k$.

Our result is the following theorem.

Theorem 1. Assume that $V$ satisfies (V1), and $K, W$, and $f$ satisfy the following:

(K1) there exist constants $a_{1}>0$ and $\gamma \in(1,2]$ such that for all $(t, q) \in[0, T] \times \mathbb{R}^{N}$

$$
K(t, 0)=0, \quad K(t, q) \geq a_{1}|q|^{\gamma} ;
$$

(K2) there exists $\theta \in(1,2]$ such that

$$
K(t, q) \leq K_{q}(t, q) q \leq \theta K(t, q), \quad \forall(t, q) \in[0, T] \times \mathbb{R}^{N} ;
$$

(W1) $W(t, 0)=0, W_{q}(t, q)=o(|q|)$, as $|q| \rightarrow 0$ uniformly for $t \in[0, T]$

(W2) there exist constants $r>2$ and $b_{1}>0$ such that

$$
W(t, q) \leq b_{1}|q|^{r}, \quad \forall(t, q) \in[0, T] \times \mathbb{R}^{N} ;
$$

(W3) there exist $\mu>1, a_{2}>0$, and $\beta(t) \in L^{1}\left(\mathbb{R}, \mathbb{R}^{+}\right)$such that $\mu>r-\gamma$ and

$$
\begin{aligned}
& W_{q}(t, q) q-2 W(t, q) \\
& \quad \geq a_{2}|q|^{\mu}-\beta(t), \quad \forall(t, q) \in[0, T] \times \mathbb{R}^{N} ;
\end{aligned}
$$

(W4) $W(t, q) /|q|^{2} \rightarrow+\infty$ as $|q| \rightarrow+\infty$ uniformly in $t \epsilon$ $[0, T]$;

(G1) $G_{j}(0)=0, g_{j}(q)=o(|q|)$, as $|q| \rightarrow 0, j=1,2, \ldots, m$;

(G2) there exists $b_{2}>0$ such that $G_{j}(q) \leq b_{2}|q|^{r}, q \in \mathbb{R}^{N}$, $j=1,2, \ldots, m$;

(G3) $g_{j}(q) q-2 G_{j}(q) \geq 0, q \in \mathbb{R}^{N} \backslash\{0\}, j=1,2, \ldots, m$;

(F1) $f \in C\left(\mathbb{R}, \mathbb{R}^{N}\right) \cap L^{2}\left(\mathbb{R}, \mathbb{R}^{N}\right) \cap L^{\mu /(\mu-1)}\left(\mathbb{R}, \mathbb{R}^{N}\right)$, $\left(\int_{-\infty}^{+\infty}|f(t)|^{2} d t\right)^{1 / 2}<\min \left\{1 / 2, a_{1}-b_{1}-M\right\}(1 / C)$, where $M=\sup \left\{G_{j}(q): j=1,2, \ldots, m,|q|=1\right\}$, $a_{1}>b_{1}+M$, and $C$ is a constant of (17). Then the system (1) and (2) possesses at least one nonzero homoclinic solution.

The rest of this paper is organized as follows. In Section 2 we present some preliminary results. Our main result's proofs are given in Section 3.

\section{Preliminaries}

Let

$$
\begin{gathered}
H_{2 k T}=\left\{q: \mathbb{R} \rightarrow \mathbb{R}^{N} \mid q, \dot{q} \in L^{2}\left(([-k T, k T]), \mathbb{R}^{N}\right),\right. \\
u(t)=u(t+2 k T), t \in \mathbb{R}\} .
\end{gathered}
$$

Then $H_{2 k T}$ is a Hilbert space with the norm defined by

$$
\|q\|_{H_{2 k T}}=\left(\int_{-k T}^{k T}\left(|\dot{q}(t)|^{2}+|q|^{2}\right) d t\right)^{1 / 2}, \quad q \in H_{2 k T} .
$$

For the norm in $L_{2 k T}^{\infty}(\mathbb{R}, \mathbb{R})$, which denotes a space of $2 k T$ periodic essentially bounded measurable functions from $\mathbb{R}$ into $\mathbb{R}^{N},\|q\|_{L_{2 k T}^{\infty}}=\operatorname{ess} \sup \{|q(t)|: t \in[-k T, k T]\}$. Next we set $\Omega_{k}=\{-k m+1,-k m+2, \ldots, 0,1,2, \ldots, k m-1, k m\}$ and define a functional $\varphi_{k}$ as

$$
\begin{aligned}
\varphi_{k}(q)= & \frac{1}{2} \eta_{k}^{2}(q)-\int_{-k T}^{k T} W d t-\sum_{j \in \Omega_{k}} G_{j}\left(q\left(t_{j}\right)\right) \\
& +\int_{-k T}^{k T} f_{k} q d t, \quad q \in H_{2 k T},
\end{aligned}
$$


where

$$
\eta_{k}(q)=\left(\int_{-k T}^{k T}|\dot{q}(t)|^{2}+2 K(t, q(t)) d t\right)^{1 / 2} .
$$

Note that $\varphi_{k}$ is Fréchet differentiable at any $q \in H_{2 k T}$, and for any $p \in H_{2 k T}$, we have

$$
\begin{aligned}
\varphi_{k}^{\prime}(q)(p)= & \lim _{h \rightarrow 0} \frac{\varphi_{k}(q+h p)-\varphi_{k}(q)}{h} \\
= & \int_{-k T}^{k T}\left(\dot{q}(t) \dot{p}(t)-V_{q}(t, q) p+f_{k} p\right) d t \\
& -\sum_{j \in \Omega_{k}} g_{j}\left(q\left(t_{j}\right)\right) p\left(t_{j}\right) .
\end{aligned}
$$

It is clear that critical points of the functional $\varphi_{k}$ are classical $2 k T$-periodic solutions of system (2) and (7).

Lemma 2 (see [2]). There is a positive constant $C$ such that for each $k \in N$ and $q \in H_{2 k T}$ the following inequality holds:

$$
\|q\|_{L_{2 k T}^{\infty}} \leq C\|q\|_{H_{2 k T}}, \quad \forall q \in H_{2 k T} .
$$

Lemma 3. $\operatorname{Set} m_{1}=\inf \left\{G_{j}(q):|q|=1, j=1,2, \ldots, m\right\}$; then for every $\zeta \in \mathbb{R} \backslash\{0\}$ and $q \in H_{2 k T} \backslash\{0\}$, we have

$$
\sum_{j \in \Omega_{k}} G_{j}\left(\zeta q\left(t_{j}\right)\right) \geq m_{1}|\zeta|^{2} \sum_{j \in \Omega_{k}}\left|q\left(t_{j}\right)\right|^{2}-2 k m m_{1} .
$$

Proof. Set $A_{k}=\left\{j \in \Omega_{k}:\left|\zeta q\left(t_{j}\right)\right| \leq 1\right\}, B_{k}=\left\{j \in \Omega_{k}\right.$ : $\left.\left|\zeta q\left(t_{j}\right)\right| \geq 1\right\}$, and $\varphi(s)=s^{2} G_{j}(q / s), j \in \Omega_{k}, s>0$. By (G3), we have

$$
\varphi^{\prime}(s)=s\left[2 G_{j}\left(\frac{q}{s}\right)-g_{j}\left(\frac{q}{s}\right) \frac{q}{s}\right] \leq 0 .
$$

So we have $G_{j}(q) \geq|q|^{2} G_{j}(q /|q|),|q| \geq 1$. If $B_{k}$ is empty, we have $q\left(t_{j}\right)=0, j \in \Omega_{k}$, which implies $\sum_{j \in \Omega_{k}} G_{j}\left(\zeta q\left(t_{j}\right)\right)=$ $0 \geq-2 \mathrm{kmm}_{1}$. Therefore, Without loss of generality, we can assume that $B_{k}$ is nonempty, and we have

$$
\begin{aligned}
\sum_{j \in \Omega_{k}} G_{j}\left(\zeta q\left(t_{j}\right)\right) & \geq \sum_{j \in B_{k}} G_{j}\left(\zeta q\left(t_{j}\right)\right) \\
& \geq \sum_{j \in B_{k}} G_{j}\left(\frac{\zeta q\left(t_{j}\right)}{\left|\zeta q\left(t_{j}\right)\right|}\right)\left|\zeta q\left(t_{j}\right)\right|^{2} \\
& \geq m_{1} \sum_{j \in B_{k}}\left|\zeta q\left(t_{j}\right)\right|^{2} \\
& \geq\left(\sum_{j \in \Omega_{k}}\left|\zeta q\left(t_{j}\right)\right|^{2}-\sum_{j \in A_{k}}\left|\zeta q\left(t_{j}\right)\right|^{2}\right) m_{1} \\
& \geq m_{1}|\zeta|^{2} \sum_{j \in \Omega_{k}}\left|q\left(t_{j}\right)\right|^{2}-2 k m m_{1} .
\end{aligned}
$$

Lemma 4 (see [30]). Let $E$ be a real Banach space and let $\varphi$ : $E \rightarrow \mathbb{R}$ be a $C^{1}$-smooth functional satisfying the Palais-Smale condition and $\varphi(0)=0$. If $\varphi$ satisfies the following conditions:

(i) there exist constants $\rho, \alpha>0$ such that $\left.\varphi\right|_{\partial B_{\rho}} \geq \alpha$,

(ii) there exists $e \in E \backslash \bar{B}_{\rho}$, such that $\varphi(e) \leq 0$, then $\varphi$ possesses a critical value $c \geq \alpha$ given by

$$
c=\inf _{g \in \Gamma \in[0,1]} \max _{S \in}(g(s)),
$$

where

$$
\Gamma=\{g \in C([0,1], E): g(0)=0, g(1)=e\} .
$$

Lemma 5 (see [2]). Let $q: \mathbb{R} \rightarrow \mathbb{R}^{N}$ be a continuous mapping such that $\dot{q} \in L_{\text {loc }}^{2}\left(\mathbb{R}, \mathbb{R}^{N}\right)$. For every $t \in \mathbb{R}$ the following inequality holds:

$$
|q(t)| \leq \sqrt{2}\left(\int_{t-1 / 2}^{t+1 / 2}\left(|q(s)|^{2}+|\dot{q}(s)|^{2}\right) d s\right)^{1 / 2} .
$$

\section{Proof of Theorem 1}

We have divided the proof of Theorem 1 into a sequence of lemmas.

Lemma 6. Assume that (V1), (K1), (K2), (W1), (W2), (W3), (W4), (G1), (G2), (G3), and (F1) are satisfied; system (2), (7) possesses a $2 k T$-periodic solution.

Proof. It is clear that $\varphi_{k}(0)=0$. It is well known that Lemma 4 holds true with the (C) condition replacing the usual (PS) condition. We say the functional $\varphi_{k}$ satisfies the (C) condition; that is, for every sequence $\left\{q_{n}\right\} \subset H_{2 k T},\left\{q_{n}\right\}$ has a convergent subsequence if $\left\{\varphi_{k}\left(q_{n}\right)\right\}$ is bounded and $\lim _{n \rightarrow \infty}\left(1+\|q\|_{H_{2 K T}}\right) \varphi_{k}^{\prime}\left(q_{n}\right)=0$.

Step 1. Pick $\left\{q_{n}\right\} \subset H_{2 k T}$ such that $\left\{\varphi_{k}\left(q_{n}\right)\right\}$ is bounded and $\lim _{n \rightarrow \infty}\left(1+\|q\|_{H_{2 k T}}\right) \varphi_{k}^{\prime}\left(q_{n}\right)=0$ then there exists a constant $C_{1}>0$ such that

$$
\begin{aligned}
C_{1} \geq & 2 \varphi_{k}\left(q_{n}\right)-\varphi_{k}^{\prime}\left(q_{n}\right)\left(q_{n}\right) \\
= & \int_{-k T}^{k T}\left[2 K\left(t, q_{n}\right)-K_{q}(t, q)\right] d t \\
& -\int_{-k T}^{k T}\left[2 W\left(t, q_{n}\right)-W_{q}\left(t, q_{n}\right) q_{n}\right] d t \\
& -\sum_{j \in \Omega_{k}}\left[2 G_{j}\left(q_{n}\left(t_{j}\right)\right)-g_{j}\left(q_{n}\left(t_{j}\right)\right) q_{n}\left(t_{j}\right)\right] \\
& +\int_{-k T}^{k T} f_{k} q_{n} d t \\
\geq & \alpha_{1} \int_{-k T}^{k T}\left|q_{n}\right|^{\mu} d t-\int_{-\infty}^{+\infty} \beta(t) d t \\
& -\left(\int_{-\infty}^{+\infty}|f|^{\mu^{\prime}} d t\right)^{1 / \mu^{\prime}}\left(\int_{-k T}^{k T}\left|q_{n}\right|^{\mu} d t\right)^{1 / \mu},
\end{aligned}
$$


where $\mu^{\prime}>1$ and $1 / \mu^{\prime}+1 / \mu=1$. (24) implies $\left(\int_{-k T}^{k T}\left|q_{n}\right|^{\mu} d t\right)^{1 / \mu}$ is bounded; that is, there exists a constant $C_{2}>0$ such that

$$
\left(\int_{-k T}^{k T}\left|q_{n}\right|^{\mu} d t\right)^{1 / \mu}<C_{2} .
$$

From (W2), (G2), (17), and (25), we have

$$
\begin{aligned}
\frac{1}{2} \eta_{k}^{2}\left(q_{n}\right)= & \varphi_{k}\left(q_{n}\right)+\int_{-k T}^{k T} W\left(t, q_{n}\right) d t \\
& -\int_{-k T}^{k T} f_{k} q_{n} d t+\sum_{j \in \Omega_{k}} G_{j}\left(q_{n}\left(t_{j}\right)\right) \\
\leq & C_{3}+b_{1}\left(C\left\|q_{n}\right\|_{H_{2 k T}}\right)^{r-\mu} \int_{-k T}^{k T}\left|q_{n}\right|^{\mu} d t \\
& +\left(\int_{-\infty}^{+\infty}|f(t)|^{\mu^{\prime}} d t\right)^{1 / \mu^{\prime}} C_{2} \\
& +b_{2}\left(C\left\|q_{n}\right\|_{H_{2 k T}}\right)^{r-\mu} \sum_{j \in \Omega_{k}}\left|q_{n}\left(t_{j}\right)\right|^{\mu} \\
\leq & C_{3}+\left(b_{1}+b_{2}\right) C_{2}^{\mu} C^{r-\mu}\left(\left\|q_{n}\right\|_{H_{2 k T}}\right)^{r-\mu} \\
& +\left(\int_{-\infty}^{+\infty}|f(t)|^{\mu^{\prime}} d t\right)^{1 / \mu^{\prime}} C_{2} .
\end{aligned}
$$

On the other hand, it follows from (K1) and (17) that

$$
\begin{aligned}
\frac{1}{2} \eta_{k}^{2}\left(q_{n}\right) \geq & \frac{1}{2} \int_{-k T}^{k T}\left[\left|\dot{q}_{n}\right|^{2}+2 a_{1}\left|q_{n}\right|^{\gamma}\right] d t \\
\geq & \frac{1}{2} \int_{-k T}^{k T}\left|\dot{q}_{n}\right|^{2} d t \\
& +a_{1}\left(C\left\|q_{n}\right\|_{H_{2 k T}}\right)^{\gamma-2} \int_{-k T}^{k T}\left|q_{n}\right|^{2} d t \\
\geq & \min \left\{\frac{1}{2}\left\|q_{n}\right\|_{H_{2 k T}}^{2}, a_{1} C^{\gamma-2}\left\|q_{n}\right\|_{H_{2 k T}}^{\gamma}\right\} .
\end{aligned}
$$

Combining (26) and (27), we obtain

$$
\begin{aligned}
\min & \left\{\frac{1}{2}\left\|q_{n}\right\|_{H_{2 k T}}^{2}, a_{1} C^{\gamma-2}\left\|q_{n}\right\|_{H_{2 k T}}^{\gamma}\right\} \\
\leq & C_{3}+\left(b_{1}+b_{2}\right) C_{2}^{\mu} C^{r-\mu}\left(\left\|q_{n}\right\|_{H_{2 k T}}\right)^{r-\mu} \\
& +\left(\int_{-\infty}^{+\infty}|f(t)|^{\mu^{\prime}}\right)^{1 / \mu^{\prime}} C_{2} .
\end{aligned}
$$

Since $r-\mu<\gamma \leq 2$, it follows that $\left\|q_{n}\right\|_{H_{2 k T}}$ is bounded. In a similar way to [21, Proposition B35], we can prove that $\left\{q_{n}\right\}$ has a convergent subsequence. So, the functional $\varphi_{k}$ satisfies the $(\mathrm{C})$ condition.

Step 2. We show that the functional $\varphi_{k}$ satisfies the assumption (i) of Lemma 4. Set $\varphi(s)=s^{2} G_{j}(q / s), j=1,2, \ldots, m$, $s>0$. By (G3), we have

$$
\varphi^{\prime}(s)=s\left[2 G_{j}\left(\frac{q}{s}\right)-g_{j}\left(\frac{q}{s}\right) \frac{q}{s}\right] \leq 0 .
$$

Hence when $0<\|q\|_{L_{2 k T}^{\infty}} \leq 1$, we have

$$
\begin{aligned}
& \sum_{j \in \Omega_{k}} G_{j}\left(q\left(t_{j}\right)\right) \\
& \leq \sum_{j \in \Omega_{k}} G_{j}\left(\frac{q\left(t_{j}\right)}{\left|q\left(t_{j}\right)\right|}\right)\left|q\left(t_{j}\right)\right|^{2} \\
& \leq M \sum_{j \in \Omega_{k}}\left|q\left(t_{j}\right)\right|^{2} \\
& \leq M\|q\|_{H_{2 k T}}^{2} .
\end{aligned}
$$

From (K1), (W2), and (30), we have

$$
\begin{aligned}
\varphi_{k}(q)= & \frac{1}{2} \eta_{k}^{2}(q)-\int_{-k T}^{k T} W(t, q) d t \\
& -\sum_{j \in \Omega_{k}} G_{j}\left(q\left(t_{j}\right)\right)+\int_{-k T}^{k T} f_{k} q d t \\
\geq & \min \left\{\frac{1}{2},\left(a_{1}-b_{1}-M\right)\right\}\|q\|_{H_{2 k T}}^{2} \\
& -\left(\int_{-\infty}^{+\infty}|f(t)|^{2} d t\right)^{1 / 2}\|q\|_{H_{2 k T} .}
\end{aligned}
$$

Set

$$
\begin{gathered}
\rho=\frac{1}{C}, \\
\alpha=\min \left\{\frac{1}{2},\left(a_{1}-b_{1}-M\right)\right\} \frac{1}{C^{2}}-\left(\int_{-\infty}^{+\infty}|f(t)|^{2} d t\right)^{1 / 2} \frac{1}{C} \\
>0 .
\end{gathered}
$$

Let $\|q\|_{H_{2 k T}}=\rho$; then $0<\|q\|_{L_{2 k T}^{\infty}} \leq 1$ and (31) gives $\varphi_{k}(q) \geq$ $\alpha>0$.

Step 3. We show that the functional $\varphi_{k}$ satisfies assumption (ii) of Lemma 4.

In order to verify (ii), we choose $\zeta \in \mathbb{R}, Q \in H_{2 T} \backslash\{0\}$ such that $Q( \pm T)=0$ and $M_{2}=\max _{|t| \leq T,|q| \leq 1} K(t, q)$. Set $h(s)=$ $s^{-\theta} K(t, s q)$. By $(\mathrm{K} 2)$, we have $h^{\prime}(s)=\left(K_{q}(t, s q) s q-\theta K(t, s q)\right) /$ $s^{\theta+1} \leq 0$. So we have

$$
K(t, q) \begin{cases}\geq|q|^{\theta} K\left(t, \frac{q}{|q|}\right), & |q| \leq 1, t \in[0, T], \\ \leq|q|^{\theta} K\left(t, \frac{q}{|q|}\right), & |q| \geq 1, t \in[0, T] .\end{cases}
$$

Define

$$
\widetilde{Q}= \begin{cases}Q, & t \in[-T, T], \\ 0, & t \in \frac{[-k T, k T]}{[-T, T]} .\end{cases}
$$


Take $A>\left(1+2 M_{2}\|Q\|_{H_{2 T}}^{2}\right) / 2 \int_{-T}^{T} Q(t) d t$. By (W4), there exists $B>0$ such that

$$
W(t, q) \geq A|q|^{2}-B, \quad t \in[0, T] \times \mathbb{R}^{N} .
$$

By (33), (34), (35), and Lemma 3, we have

$$
\begin{aligned}
\varphi_{k}(\zeta \widetilde{Q})= & \frac{1}{2} \eta_{1}^{2}(\zeta Q)-\int_{-T}^{T} W(t, \zeta Q) d t \\
& -\sum_{j \in \Omega_{1}} G_{j}\left(\zeta Q\left(t_{j}\right)\right)+\zeta \int_{-T}^{T} f_{1} Q d t \\
\leq & \frac{1}{2}\left(1+2 M_{2}\right)|\zeta|^{2}\|Q\|_{H_{2 T}}^{2}-A|\zeta|^{2} \int_{-T}^{T}|Q(t)|^{2} d t \\
& +2\left(M_{2} T+T B+m m_{2}\right) \\
& -m_{1}|\zeta|^{2} \sum_{j \in \Omega_{1}}\left|Q\left(t_{j}\right)\right|^{2} \\
& +|\zeta|\left(\int_{-T}^{T}\left|f_{1}\right|^{2} d t\right)^{1 / 2}\left(\int_{-T}^{T}|Q|^{2} d t\right)^{1 / 2} \\
\leq & |\zeta|^{2}\left(\frac{1+2 M_{2}}{2}\|Q\|_{H_{2 T}}^{2}-A \int_{-T}^{T}|Q(t)|^{2} d t\right) \\
& +2\left(M_{2} T+T B+m m_{1}\right) \\
& +|\zeta|\left(\int_{-T}^{T}\left|f_{1}\right|^{2} d t\right)^{1 / 2}\left(\int_{-T}^{T}|Q|^{2} d t\right)^{1 / 2} .
\end{aligned}
$$

Clearly, $\varphi_{k}(\zeta q) \rightarrow-\infty$ as $|\zeta| \rightarrow+\infty$, so (ii) holds. By Lem-ma $4, \varphi_{k}$ possesses a critical value $c_{k} \geq \alpha>0$. Let $q_{k}$ denote the corresponding critical point of $\varphi_{k}$ on $H_{2 k T}$; that is,

$$
\varphi_{k}\left(q_{k}\right)=c_{k}, \quad \varphi_{k}^{\prime}\left(q_{k}\right)=0 .
$$

Hence the system (2), (7) possesses a nontrivial $2 k T$-periodic solution $q_{k}$.

Lemma 7. Let $\left\{q_{k}\right\}$ be the sequence given by (37). Then there exist a subsequence $\left\{q_{j, j}\right\}$ of $\left\{q_{k}\right\}$ and a function $q_{0} \in W_{l o c}^{1,2} \cap$ $L_{\text {loc }}^{\infty}\left(\mathbb{R}, \mathbb{R}^{\mathbb{N}}\right)$ such that $\left\{q_{j, j}\right\}$ converges to $q_{0}$ weakly in $W_{\text {loc }}^{1,2}$ and strongly in $L_{\text {loc }}^{\infty}\left(\mathbb{R}, \mathbb{R}^{\mathbb{N}}\right)$.

Proof. We assert that there is a constant $M_{3}>0$ independent of $k$ such that

$$
\left\|q_{k}\right\|_{H_{2 k T}} \leq M_{3} .
$$

Let $e_{1} \in H_{2 T} \backslash\{0\}$ such that $e_{1}( \pm T)=0, e_{1}\left(t_{k}\right) \neq 0$ for some $t_{k} \in$ $(-T, T)$, and $\varphi_{1}\left(e_{1}\right) \leq 0$. Define

$$
e_{k}= \begin{cases}e_{1}, & |t| \leq T, \\ 0, & T \leq|t| \leq k T .\end{cases}
$$

We extend $e_{k}$ in the way of $2 k T$-periodic to $\mathbb{R}$. For simplicity, we also note it again by $e_{k}$. It is clear that $e_{k} \in H_{2 k T}$ and $\varphi_{k}\left(e_{k}\right)=\varphi_{1}\left(e_{1}\right) \leq 0$. Define $g_{k}:[0,1] \rightarrow H_{2 k T}$ by $g_{k}(s)=$ $s e_{k}$ for $s \in[0,1]$. Then, we have

$$
c_{k} \leq \max _{s \in[0,1]} \varphi_{k}\left(g_{k}(s)\right)=\max _{s \in[0,1]} \varphi_{1}\left(g_{1}(s)\right) \equiv c_{0},
$$

independently of $k$. The rest detailed argument is similar to the proof of Step 1 in Lemma 6 and we thus omit it here.

Hence, $\left\{q_{k}\right\}$ is a bounded sequence in $W^{1,2}\left((-T, T), \mathbb{R}^{N}\right)$ and we may pick a subsequence $\left\{q_{1, k}\right\}$ such that $\left\{q_{1, k}\right\}$ converges weakly in $W^{1,2}\left((-T, T), \mathbb{R}^{N}\right)$ and strongly in $L^{\infty}\left((-T, T), \mathbb{R}^{N}\right)$. Next $\left\{q_{1, k}\right\}$ is a bounded sequence in $W^{1,2}\left((-2 T, 2 T), \mathbb{R}^{N}\right)$, so we may pick a subsequence $\left\{q_{2, k}\right\}$ such that $\left\{q_{2, k}\right\}$ converges weakly in $W^{1,2}\left((-2 T, 2 T), \mathbb{R}^{N}\right)$ and strongly in $L^{\infty}\left((-2 T, 2 T), \mathbb{R}^{N}\right)$. We can repeat this process and obtain, for any positive integer $n$, a sequence $\left\{q_{n, k}\right\}$ which converges weakly in $W^{1,2}\left((-n T, n T), \mathbb{R}^{N}\right)$ and strongly in $L^{\infty}\left((-n T, n T), \mathbb{R}^{N}\right)$, and

$$
\left\{q_{k}\right\} \supset\left\{q_{1, k}\right\} \supset\left\{q_{2, k}\right\} \supset \cdots \supset\left\{q_{n, k}\right\} \supset \cdots .
$$

Therefore, for any positive integer $n$, the sequence $\left\{q_{k, k}\right\}$ converges weakly in $W^{1,2}\left((-n T, n T), \mathbb{R}^{N}\right)$ and strongly in $L^{\infty}\left((-n T, n T), \mathbb{R}^{N}\right)$. Hence there exists a function $q \in$ $W_{\text {loc }}^{1,2}\left(\mathbb{R}, \mathbb{R}^{N}\right) \cap L_{\text {loc }}^{\infty}\left(\mathbb{R}, \mathbb{R}^{N}\right)$ such that the sequence $\left\{q_{k, k}\right\}$ converges weakly in $q \in W_{\text {loc }}^{1,2}\left(\mathbb{R}, \mathbb{R}^{N}\right)$ and strongly in $L_{\text {loc }}^{\infty}\left(\mathbb{R}, \mathbb{R}^{N}\right)$.

Lemma 8. The function $q_{0}$ determined by Lemma 7 is a nonzero homoclinic solution of the system (1), (2).

Proof. The proof will be divided into four steps.

Firstly, we show that $q_{0}$ is a solution of the system (1), (2). Here, for simplicity, we denote $\left\{q_{k, k}\right\}$ by $\left\{q_{k}\right\}$. For any given interval $(a, b) \subset(-k T, k T)$ and any $p \in W_{0}^{1,2}\left((a, b), \mathbb{R}^{N}\right)$, define

$$
p_{1}= \begin{cases}p, & t \in(a, b), \\ 0, & t \in(-k T, k T) \backslash(a, b),\end{cases}
$$

so for any $p \in W_{0}^{1,2}\left((a, b), \mathbb{R}^{N}\right)$, we have

$$
\begin{aligned}
0= & \varphi_{k}^{\prime}\left(q_{k}\right) p_{1} \\
= & \int_{a}^{b}\left(\dot{q}_{k} \dot{p}-V_{q}\left(t, q_{k}\right) p+f_{k} p\right) d t \\
& +\sum_{t_{j} \in(a, b)} g_{j}\left(q_{k}\left(t_{j}\right)\right) p\left(t_{j}\right) .
\end{aligned}
$$


Therefore, one has

$$
\begin{gathered}
\int_{a}^{b}\left(\dot{q}_{0} \dot{p}-V_{q}\left(t, q_{0}\right) p+f p\right) d t+\sum_{t_{j} \in(a, b)} g_{j}\left(q_{0}\left(t_{j}\right)\right) p\left(t_{j}\right) \\
=\lim _{k \rightarrow+\infty}\left(\int_{a}^{b}\left(\dot{q}_{k} \dot{p}-V_{q}\left(t, q_{k}\right) p+f_{k} p\right) d t\right. \\
\left.+\sum_{t_{j} \in(a, b)} g_{j}\left(q_{k}\left(t_{j}\right)\right) p\left(t_{j}\right)\right)
\end{gathered}
$$

$=0$.

The remained detailer argument is similar to the proof of Lemma 2.5 in [13] and we thus omit it here, so $q_{0}(t)$ is a solution of system (1) and (2).

Secondly we show that $q_{0}(t) \rightarrow 0$, as $t \rightarrow \pm \infty .\left\{q_{k}\right\}$ is weak continuity, so it is weak lower semicontinuity. One has

$$
\begin{aligned}
& \int_{-\infty}^{+\infty}\left(\left|q_{0}\right|^{2}+\left|\dot{q}_{0}\right|^{2}\right) d t \\
& \quad=\lim _{k \rightarrow+\infty} \int_{-k T}^{k T}\left(\left|q_{0}\right|^{2}+\left|\dot{q}_{0}\right|^{2}\right) d t \\
& \quad \leq \lim _{k \rightarrow+\infty} \lim _{j \rightarrow+\infty} \inf \int_{-k T}^{+k T}\left(\left|q_{j}\right|^{2}+\left|\dot{q}_{j}\right|^{2}\right) d t \\
& \leq M_{3}^{2},
\end{aligned}
$$

and so

$$
\int_{|t| \geq r}\left(\left|q_{0}\right|^{2}+\left|\dot{q}_{0}\right|^{2}\right) d t \rightarrow 0, \quad \text { as } r \longrightarrow+\infty .
$$

By (23) and (46), we obtain $q_{0}(t) \rightarrow 0$, as $t \rightarrow \pm \infty$.

Thirdly, we prove that $\dot{q}_{0}\left(t^{ \pm}\right) \rightarrow 0$ as $t \rightarrow \pm \infty$. We have proved $q_{0}(t)$ is a solution of system (1) and (2), so we have

$$
\begin{aligned}
& \int_{t_{j-1}}^{t_{j}}\left|\ddot{q}_{0}(s)\right|^{2} d s \\
& \quad=\int_{t_{j-1}}^{t_{j}}\left(-V_{q}\left(s, q_{0}(s)\right)+f(s)\right)^{2} d s \\
& \quad \leq 2 \int_{t_{j-1}}^{t_{j}}\left(\left|V_{q}\left(s, q_{0}(s)\right)\right|^{2}+|f(s)|^{2}\right) d s .
\end{aligned}
$$

By (V1), (K1), and (W1), one has $V_{q}(t, 0)=-K_{q}(t, 0)+$ $W_{q}(t, 0)=0$. Hence $\int_{t_{j-1}}^{t_{j}}\left|\ddot{q}_{0}(s)\right|^{2} d s \rightarrow 0$ as $j \rightarrow \pm \infty$. By $(23)$, one has

$$
\begin{aligned}
\left|\dot{q}_{0}(t)\right|^{2} \leq & 2 \int_{t_{j-1}}^{t_{j}}\left(\left|\dot{q}_{0}(s)\right|^{2}+\left|\ddot{q}_{0}(s)\right|^{2}\right) d s \\
\leq & 2 \int_{t_{j-1}}^{t_{j}}\left(\left|q_{0}(s)\right|^{2}+\left|\dot{q}_{0}(s)\right|^{2}\right) d s \\
& +2 \int_{t_{j-1}}^{t_{j}}\left|\ddot{q}_{0}(s)\right|^{2} d s, \quad t \in\left(t_{j-1}, t_{j}\right) .
\end{aligned}
$$

Therefore one has $\dot{q}_{0}\left(t^{ \pm}\right) \rightarrow 0$ as $t \rightarrow \pm \infty$.
Finally, we show $q_{0} \neq 0$ when $f \equiv 0$. Since $0=t_{0}<t_{1}<$ $\cdots<t_{m}=T, t_{j+m}=t_{j}+T, j \in \mathbb{Z}$, we can let $\underline{\delta}=\min _{j \in \mathbb{Z}}\left\{t_{j}-\right.$ $\left.t_{j-1}\right\}$ and $\bar{\delta}=\max _{j \in \mathbb{Z}}\left\{t_{j}-t_{j-1}\right\}$. By Hölder inequality and $q_{k}\left(t_{j}\right)=q_{k}(\tau)+\int_{\tau}^{t_{j}} \dot{q}_{k}(s) d s, \tau \in\left[t_{j-1}, t_{j}\right], j \in \Omega_{k}$, we have

$$
\begin{aligned}
& \sum_{j \in \Omega_{k}}\left|q_{k}\left(t_{j}\right)\right|^{2} \leq \frac{1}{\underline{\delta}} \sum_{j \in \Omega_{k}} \int_{t_{j-1}}^{t_{j}}\left|q_{k}\left(t_{j}\right)\right|^{2} d \tau \\
&=\frac{1}{\underline{\delta}} \sum_{j \in \Omega_{k}} \int_{t_{j-1}}^{t_{j}}\left(q_{k}(\tau)+\int_{\tau}^{t_{j}} \dot{q}_{k}(s) d s\right)^{2} d \tau \\
& \leq \frac{2}{\underline{\delta}} \sum_{j \in \Omega_{k}} \int_{t_{j-1}}^{t_{j}}\left(\left|q_{k}(\tau)\right|^{2}+\left|\int_{\tau}^{t_{j}} \dot{q}_{k}(s) d s\right|^{2}\right) d \tau \\
& \leq \frac{2}{\underline{\delta}} \int_{-k T}^{k T}\left|q_{k}(\tau)\right|^{2} d \tau \\
& \quad+\frac{2}{\delta} \sum_{j \in \Omega_{k}} \int_{t_{j-1}}^{t_{j}}\left(\left(t_{j}-\tau\right) \int_{\tau}^{t_{j}}\left|\dot{q}_{k}(s)\right|^{2} d s\right) d \tau \\
& \leq \frac{2}{\delta} \int_{-k T}^{k T}\left|q_{k}(\tau)\right|^{2} d \tau+\frac{2 \bar{\delta}^{2}}{\underline{\delta}} \int_{-k T}^{k T}\left|\dot{q}_{k}(\tau)\right|^{2} d \tau \\
& \leq \max \left\{1, \bar{\delta}^{2}\right\} \frac{2}{\delta}\left\|q_{k}\right\|_{H_{2 k T}}^{2}
\end{aligned}
$$

Let $M_{4}=\max \left\{1, \bar{\delta}^{2}\right\}(2 / \underline{\delta})>0$, which is a constant independent of $k$.

It is clearly that $q_{k}(t+j T), j \in \mathbb{Z}$, is a $2 k T$ periodic solution of (2), (7). So we can assume the maximum of $q_{k}$ occurs in $[-T, T]$. Now we assume $q_{0}=0$, so there is $\left\|q_{k}\right\|_{L_{2 k T}^{\infty}}=$ $\max _{t \in[-T, T]}\left|q_{k}\right| \rightarrow 0$ as $k \rightarrow+\infty$; therefore there exists integer $N_{1}>0$ such that $\left\|q_{k}\right\|_{L_{2 k T}^{\infty}}<1$. Combining (G1), there exists an integer $N_{2}>0$ such that when $k>N_{2}$, one has

$$
\frac{\left|g_{j}\left(q_{k}\left(t_{j}\right)\right)\right|}{\left|q_{k}\left(t_{j}\right)\right|} \leq \frac{1}{2 M_{4}} \min \left\{1, a_{1}\right\}, \quad j \in \mathbb{Z} .
$$

By (49) and (50), when $k>N_{2}$, one has

$$
\begin{aligned}
& \sum_{j \in \Omega_{k}} g_{j}\left(q_{k}\left(t_{j}\right)\right) q_{k}\left(t_{j}\right) \\
& \quad \leq \sum_{j \in \Omega_{k}} \frac{\left|g_{j}\left(q_{k}\left(t_{j}\right)\right)\right|}{\left|q_{k}\left(t_{j}\right)\right|}\left|q_{k}\left(t_{j}\right)\right|^{2} \\
& \quad \leq \frac{1}{2} \min \left\{1, a_{1}\right\}\left\|q_{k}\right\|_{H_{2 k T}}^{2} .
\end{aligned}
$$

Define a function $Y: \mathbb{R}^{+} \rightarrow \mathbb{R}^{+}$by

$$
Y(s)= \begin{cases}0, & s=0, \\ \max _{t \in[0, T],|\xi| \leq s} \frac{\left|\xi W_{q}(t, \xi)\right|}{|\xi|^{2}}, & s>0 .\end{cases}
$$


It is clear that $Y \in C\left(\mathbb{R}^{+}, \mathbb{R}^{+}\right)$and is monotone nondecreasing, so we have

$$
\frac{\left|q_{k} W_{q}\left(t, q_{k}(t)\right)\right|}{\left|q_{k}(t)\right|^{2}} \leq Y\left(\left\|q_{k}\right\|_{L_{[-k T, k T]}^{\infty}}\right), \quad t \in[-k T, k T] .
$$

Hence we have

$$
\begin{aligned}
& \int_{-k T}^{k T}\left|q_{k} W_{q}\left(t, q_{k}(t)\right)\right| d t \\
& \quad \leq Y\left(\left\|q_{k}\right\|_{L_{[-k T, k T]}^{\infty}}\right) \int_{-k T}^{k T}\left|q_{k}(t)\right|^{2} d t \\
& \quad \leq Y\left(\left\|q_{k}\right\|_{L_{[-k T, k T]}^{\infty}}\right)\left\|q_{k}\right\|_{H_{2 k T}}^{2} .
\end{aligned}
$$

Since $f \equiv 0, q_{k}$ is a solution of the system (1) and (2), so when $k>\max \left\{N_{1}, N_{2}\right\}$, we have

$$
\begin{gathered}
\int_{-k T}^{k T} q_{k} W_{q}\left(t, q_{k}(t)\right) d t+\sum_{j \in \Omega_{k}} g_{j}\left(q_{k}\left(t_{j}\right)\right) q_{k}\left(t_{j}\right) \\
\quad \geq \min \left\{1, a_{1}\right\}\left\|q_{k}\right\|_{H_{2 k T}}^{2} .
\end{gathered}
$$

Combining (51), (54), and (55) we have

$$
\begin{gathered}
Y\left(\left\|q_{k}\right\|_{L_{[-k T, k T]}^{\infty}}\right)\left\|q_{k}\right\|_{H_{2 k T}}^{2}+\frac{1}{2} \min \left\{1, a_{1}\right\}\left\|q_{k}\right\|_{H_{2 k T}}^{2} \\
\geq \min \left\{1, a_{1}\right\}\left\|q_{k}\right\|_{H_{2 k T}}^{2} .
\end{gathered}
$$

Hence, we have

$$
Y\left(\left\|q_{k}\right\|_{L_{[-k T, k T]}^{\infty}}\right) \geq \frac{1}{2} \min \left\{1, a_{1}\right\}>0 .
$$

By the property of the function $Y$, there exists a constant $M_{5}>$ 0 such that $\left\|q_{k}\right\|_{L_{[-k T, k T]}^{\infty}} \geq M_{5}>0$. This is a contradiction. Hence the system (1), (2) has a nontrivial homoclinic solution even if $f \equiv 0$.

Next, we give an example to illustrate our main result.

Example 9. Let

$$
\begin{gathered}
K(t, q)=(4+\sin t)|q|^{4 / 3}, \\
W(t, q)=(2+\cos t)|q|^{2} \ln (1+|q|), \\
G_{j}\left(q\left(t_{j}\right)\right)=\left|\sin \left(\frac{t_{j}}{2}\right)\right| q^{r}\left(t_{j}\right), \\
g_{j}\left(q\left(t_{j}\right)\right)=r\left|\sin \left(\frac{t_{j}}{2}\right)\right| q^{r-1}\left(t_{j}\right), \quad f(t)=0,
\end{gathered}
$$

where $r>2, t_{j}=2 \pi j / m, j \in \mathbb{N}$. It is easy to verify that $K, W$, $G_{j}, g_{j}$, and $f$ satisfy conditions (V1), (K1), (K2), (W1), (W2), (W3), (W4), (G1), (G2), (G3), and (F1). So, system (1), (2) with $K, W, G_{j}, g_{j}$, and $f$ as in (58) has a nontrivial homoclinic solution.

\section{Acknowledgments}

The authors are very grateful to the referees for their very helpful comments and suggestions, which greatly improved the presentation of this paper. This work is supported by the Hunan Provincial Natural Science Foundation of China (no. 11JJ3012) and Major Project of Science Research Fund of Education Department in Hunan (no. 11A095).

\section{References}

[1] P. H. Rabinowitz, "Homoclinic orbits for a class of Hamiltonian systems," Proceedings of the Royal Society of Edinburgh. Section A, vol. 114, no. 1-2, pp. 33-38, 1990.

[2] M. Izydorek and J. Janczewska, "Homoclinic solutions for a class of the second order Hamiltonian systems," Journal of Differential Equations, vol. 219, no. 2, pp. 375-389, 2005.

[3] R. P. Agarwal and D. O’Regan, "A multiplicity result for second order impulsive differential equations via the Leggett Williams fixed point theorem," Applied Mathematics and Computation, vol. 161, no. 2, pp. 433-439, 2005.

[4] R. P. Agarwal, D. Franco, and D. O’Regan, "Singular boundary value problems for first and second order impulsive differential equations," Aequationes Mathematicae, vol. 69, no. 1-2, pp. 8396, 2005.

[5] J. Chu and J. J. Nieto, "Impulsive periodic solutions of firstorder singular differential equations," Bulletin of the London Mathematical Society, vol. 40, no. 1, pp. 143-150, 2008.

[6] Z. He and X. He, "Monotone iterative technique for impulsive integro-differential equations with periodic boundary conditions," Computers \& Mathematics with Applications, vol. 48, no. 1-2, pp. 73-84, 2004.

[7] J. J. Nieto and D. O'Regan, "Variational approach to impulsive differential equations," Nonlinear Analysis: Real World Applications, vol. 10, no. 2, pp. 680-690, 2009.

[8] D. Qian and X. Li, "Periodic solutions for ordinary differential equations with sublinear impulsive effects," Journal of Mathematical Analysis and Applications, vol. 303, no. 1, pp. 288-303, 2005.

[9] P. H. Rabinowitz, Minimax Methods in Critical Point Theory with Applications to Differential Equations, vol. 65 of CBMS Regional Conference Series in Mathematics, American Mathematical Society, Washington, DC, USA, 1986.

[10] I. Rachůnková and M. Tvrdý, "Non-ordered lower and upper functions in second order impulsive periodic problems," Dynamics of Continuous, Discrete \& Impulsive Systems. Series A, vol. 12, no. 3-4, pp. 397-415, 2005.

[11] J. Sun, H. Chen, and L. Yang, "Variational methods to fourthorder impulsive differential equations," Journal of Applied Mathematics and Computing, vol. 35, no. 1-2, pp. 323-340, 2011.

[12] J. Shen and W. Wang, "Impulsive boundary value problems with nonlinear boundary conditions," Nonlinear Analysis: Theory, Methods \& Applications, vol. 69, no. 11, pp. 4055-4062, 2008.

[13] Y. Tian and W. Ge, "Applications of variational methods to boundary-value problem for impulsive differential equations," Proceedings of the Edinburgh Mathematical Society, vol. 51, no. 2, pp. 509-527, 2008.

[14] Y. Tian, J. Wang, and W. Ge, "Variational methods to mixed boundary value problem for impulsive differential equations with a parameter," Taiwanese Journal of Mathematics, vol. 13, no. 4, pp. 1353-1370, 2009. 
[15] J. Xie and Z. Luo, "Multiple solutions for a second-order impulsive Sturm-Liouville equation," Abstract and Applied Analysis, vol. 2013, Article ID 527082, 6 pages, 2013.

[16] J. Xie and Z. Luo, "Solutions to a boundary value problem of a fourth-order impulsive differential equation," Boundary Value Problems, vol. 2013, 154 pages, 2013.

[17] W. Zuo, D. Jiang, D. O'Regan, and R. P. Agarwal, "Optimal existence conditions for the periodic delay $\phi$-Laplace equation with upper and lower solutions in the reverse order," Results in Mathematics, vol. 44, no. 3-4, pp. 375-385, 2003.

[18] H. Zhang and Z. Li, "Periodic and homoclinic solutions generated by impulses," Nonlinear Analysis: Real World Applications, vol. 12, no. 1, pp. 39-51, 2011.

[19] H. Zhang and Z. Li, "Variational approach to impulsive differential equations with periodic boundary conditions," Nonlinear Analysis: Real World Applications, vol. 11, no. 1, pp. 67-78, 2010.

[20] Z. Zhang and R. Yuan, "An application of variational methods to Dirichlet boundary value problem with impulses," Nonlinear Analysis: Real World Applications, vol. 11, no. 1, pp. 155-162, 2010.

[21] X. Lv and S. Lu, "Homoclinic orbits for a class of second-order Hamiltonian systems without a coercive potential," Journal of Applied Mathematics and Computing, vol. 39, no. 1-2, pp. 121130, 2012.

[22] P. D. Makita, "Homoclinic orbits for second order Hamiltonian equations in $\mathbb{R}$," Journal of Dynamics and Differential Equations, vol. 24, no. 4, pp. 857-871, 2012.

[23] X. H. Tang and L. Xiao, "Homoclinic solutions for a class of second-order Hamiltonian systems," Nonlinear Analysis: Theory, Methods \& Applications, vol. 71, no. 3-4, pp. 1140-1152, 2009.

[24] M. Winkler, "Spatially monotone homoclinic orbits in nonlinear parabolic equations of super-fast diffusion type," Mathematische Annalen, vol. 355, no. 2, pp. 519-549, 2013.

[25] X. Zhang and X. Tang, "Subharmonic solutions for a class of non-quadratic second order Hamiltonian systems," Nonlinear Analysis: Real World Applications, vol. 13, no. 1, pp. 113-130, 2012.

[26] Z. Zhou, J. Yu, and Y. Chen, "Homoclinic solutions in periodic difference equations with saturable nonlinearity," Science China Mathematics, vol. 54, no. 1, pp. 83-93, 2011.

[27] V. Coti-Zelati, I. Ekeland, and E. Séré, "A variational approach to homoclinic orbits in Hamiltonian systems," Mathematische Annalen, vol. 288, no. 1, pp. 133-160, 1990.

[28] P.-L. Lions, "The concentration-compactness principle in the calculus of variations. The locally compact case. I," Annales de l'Institut Henri Poincaré, vol. 1, no. 2, pp. 109-145, 1984.

[29] H. Hofer and K. Wysocki, "First order elliptic systems and the existence of homoclinic orbits in Hamiltonian systems," Mathematische Annalen, vol. 288, no. 3, pp. 483-503, 1990.

[30] A. Ambrosetti and P. H. Rabinowitz, "Dual variational methods in critical point theory and applications," Journal of Functional Analysis, vol. 14, no. 4, pp. 349-381, 1973. 


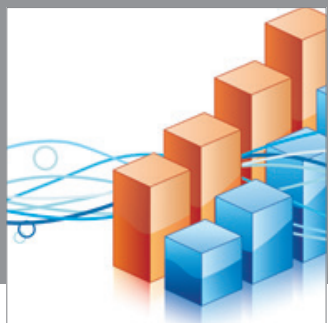

Advances in

Operations Research

mansans

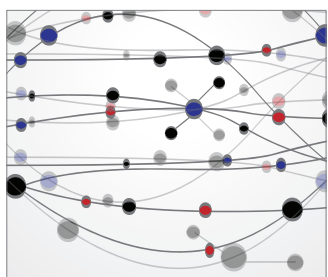

The Scientific World Journal
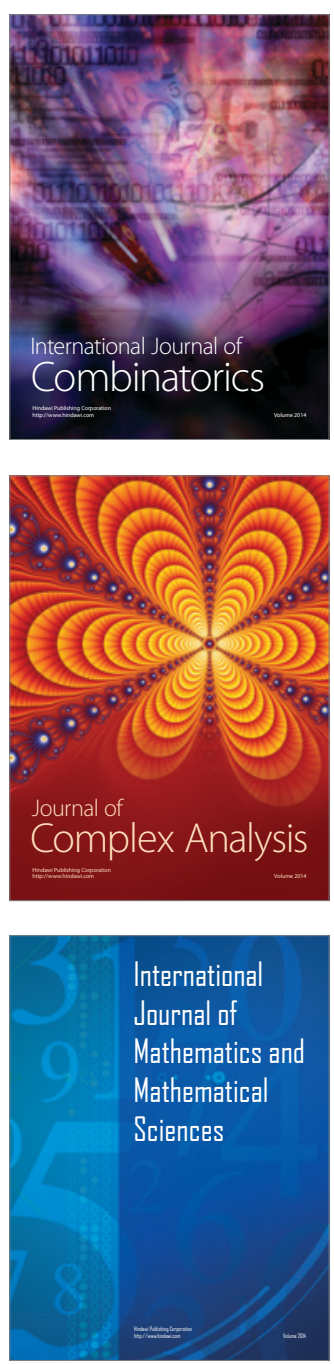
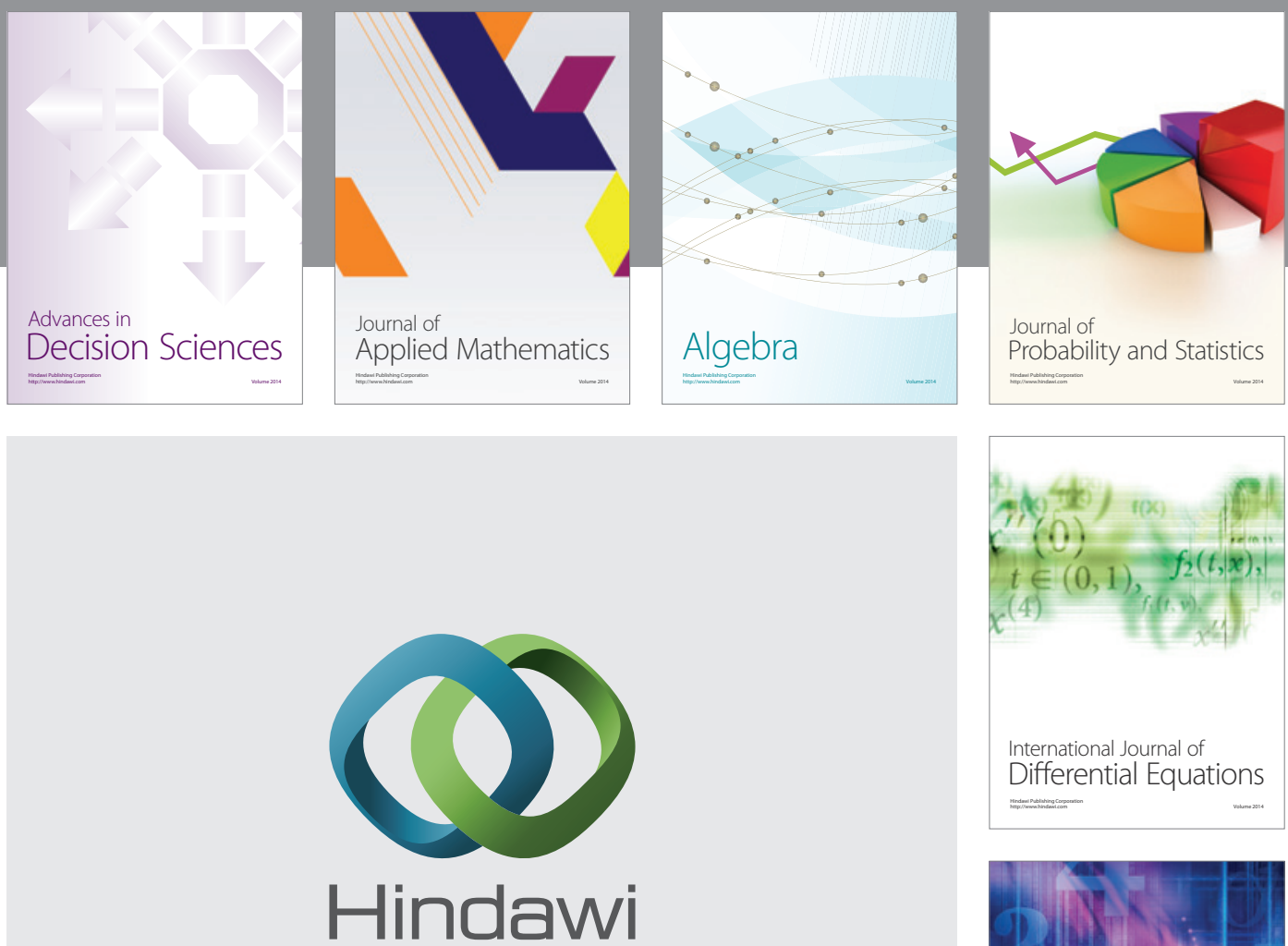

Submit your manuscripts at http://www.hindawi.com
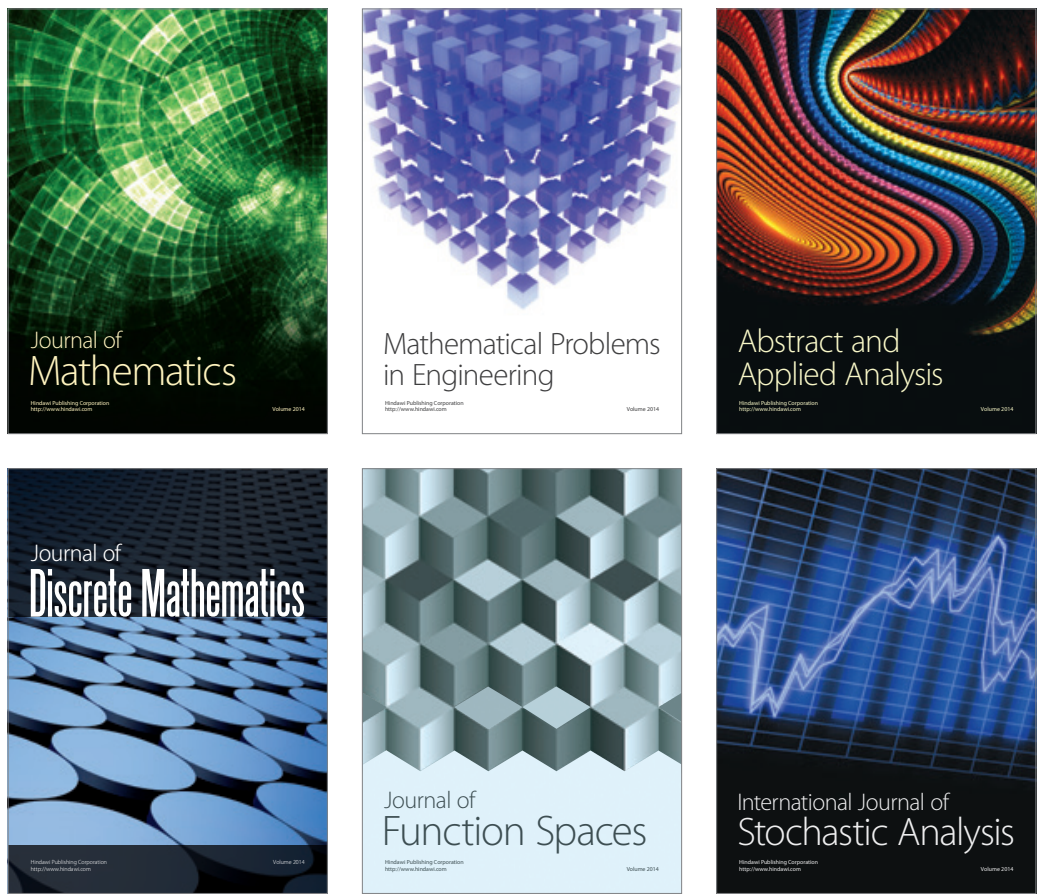

Journal of

Function Spaces

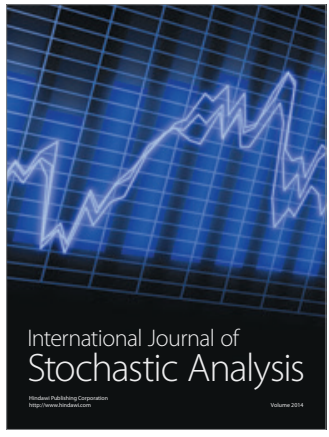

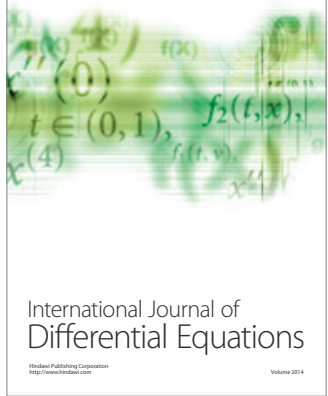
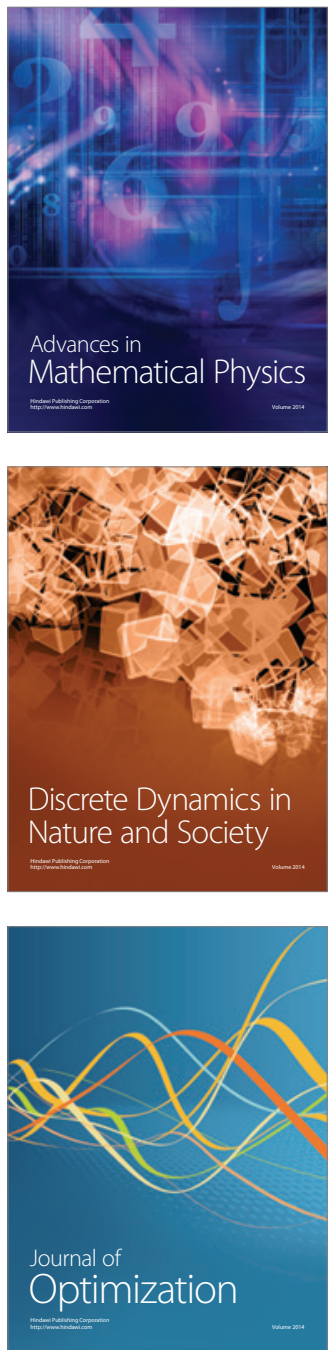